\title{
Gamification: focus on the strategies being implemented in interventions: a systematic review protocol
}

\author{
Sílvia Lopes* ${ }^{*}$, Armanda Pereira, Paula Magalhães, André Oliveira and Pedro Rosário
}

\begin{abstract}
Objective: Gamification broadly refers to the use of game design elements in non-game contexts with the goal of promoting users' engagement. Gamification strategies appear as an advantageous tool to increase the motivation and involvement of users. The purpose of this systematic review is to identify studies using gamification strategies in distinct intervention contexts and to describe their impact in each type of intervention. Thus, the focus is on the construct (gamification) rather than on a particular area or population.

Results: To achieve this goal, Scopus, IEEE, Web of Science, MEDLINE, ERIC, and PsycINFO databases will be used to gather data for the systematic review. Both the research and report of results will be based on Cochrane's recommendations and PRISMA guidelines. Data, including the assessment on the quality of the articles, will be conducted by two members of the team independently. Findings will be reported narratively. The ethical approval is not required as the research does not involve data collection. Findings will be submitted to a peer-review journal and the results will be presented on international congresses. Future reviews could consider investigating particular areas of intervention in which gamification strategies are being employed.
\end{abstract}

Trial registration Systematic Review Registration: PROSPERO CRD42017070508

Keywords: Gamification, Intervention, Systematic review, Gamify, Game elements

\section{Introduction}

The term gamification was originally coined in 2008 [1] within the digital media industry, and broadly refers to the use of game design elements in non-game contexts with the goal of promoting users' engagement [1]. From 2010 onwards, interest in the subject has spread into distinct domains of knowledge (e.g., education [e.g., 2], health [e.g., 3] and business management [e.g., 4], and the term has been referred in scientific publications, academia, publicity, industry and health [e.g., 1, 5-7]. The purposes of implementing gamification in such contexts were manifold, such as binding individuals to the tasks at hand, increasing productivity and financial profit, and increasing overall interest in the task $[1,6]$.

\footnotetext{
*Correspondence: silvia.lopes.psi@gmail.com
}

School of Psychology, University of Minho, Campus de Gualtar,

4710-057 Braga, Portugal
Aiming to promote engagement and motivation in the task at hand $[1,8]$, gamification works by transferring specific features of games to systems working in non-traditional gaming contexts (e.g., rehabilitation) $[1,6]$. In fact, gamification has been analyzed in light of psychological theories of motivation, such as the theory of intrinsic motivation [e.g., 9]. Accordingly, gamified systems present motivational characteristics, such as immediate feedback, indications regarding individual progress or goal setting attainment (through scores, emblems, competitions), relational support, and social feedback and autonomy (to control avatars, and decision making) [1]. Thus, especially for younger populations, the components of gamification may improve the intervention's intrinsic interest, promote learning opportunities, enhance the degree and level of participants' engagement, and foster their behavior and lifestyle [3,10-12]. This is accomplished 
by involving users through strategies as follows: setting short, medium, and long terms goals, using challenges to display interest, and providing rewards (e.g., badges) for completing actions or achieving scores within a specific grade range on an activity [13, 14]. For instance, in the educative context, gamification of learning may involve "the use of game elements, including action language, assessment, conflict/challenge, control, environment, game fiction, human interaction, immersion, and rules/goals, to facilitate learning and related outcomes" [15, p. 6].

Unequivocally, the use of game-design elements constitutes a unique opportunity to engage participants in specific activities (e.g., rehabilitation or clinical interventions) [16-18]. Regarding the use of gamification in the context of an intervention, two main categories can be distinguished regarding their purpose: (i) gamification for development, i.e. the process of using gamification to build, for example, a new tool or platform with specific purposes; and (ii) gamification for enhancement, i.e. the practice of gamification to increase an already well-established activity [19]. However, implementing a gamification approach within interventions (e.g., rehabilitation or educational focused) is considered a difficult task, and it is listed within the group of complex interventions [19]. All considered, it is necessary to further understand the phenomena and to help practitioners [19].

Previous reviews on this subject addressed important aspects of gamification strategies in different areas of knowledge (e.g., healthcare) and have highlighted that gamifying experiences translate into improvements in psychological and behavioral outcomes [6]. However, none have enquired about the game components and assessment strategies used or their relationship with the efficacy of the interventions. Besides, embedding gamification strategies into interventions is becoming ubiquitous; therefore, it is critical to systematize available information on which elements are being incorporated, and examine how they add up to interventions-as-usual effectiveness regardless of the purpose of the intervention or of its target population. Despite the significant increase in studies using gamification strategies in different areas, little is known about the effectiveness of the use of gamification [8]. Thus, it is important to further understand the phenomenon in depth. Accordingly, the objective of this qualitative review is to understand the phenomenon of gamification, and its effectiveness, regardless of the domain of the intervention (e.g., health, academic) in which the gamification strategies were employed.

\section{Main text}

The present protocol was developed and submitted by the authors for registration in PROSPERO prior to the beginning of the research. The protocol followed the procedures considered in the writing of systematic reviews, namely Cochrane and PRISMA guidelines. All studies including interventions with gamification were considered for review. For a better understanding, authors considered intervention as any action on a given population with a therapeutic purpose and gamification as "the use of game design elements in nongame contexts" [1].

This systematic review protocol is in line with the items of the Preferred Networks for Systematic Review Initiatives and Meta-Analysis Protocols (PRISMAP) [20] (see Additional file 1). The current systematic review will follow the guidelines of the Cochrane Collaboration, namely the search strategy, identification, collection and characterization of eligible papers, and the organization of results. The Cochrane Collaboration method is detailed in the Cochrane Handbook for Systematic Reviews of Intervention [21]. Moreover, from the data abstraction phase onwards, PRISMA [22] guidelines will be followed. The PRISMA Statement provides an evidence-based 27-item checklist (e.g., focused on methodology) for reporting in systematic reviews and meta-analyses.

\section{Eligibility criteria}

To determine the ways in which gamification has been used in intervention, a systematic approach will be conducted to retrieve relevant papers from the literature. Articles will be excluded from the review when: (i) they are not empirical studies; (ii) they are not written in Spanish, Portuguese or English languages; and (iii) they are not focused on gamification strategies. The following study characteristics will guide the process of inclusion and exclusion of articles. Study characteristics are described in Table 1.

\section{Outcomes}

The systematic review will allow authors to identify the gamification elements most used, the characteristics of the participants, the type and objectives of the intervention, the environment where the intervention is carried out, the assessment performed, and the effectiveness of these interventions.

\section{Information sources}

This systematic literature review aims to identify articles containing information about interventions using gamification. For this purpose, searches will be 
Table 1 Description of study characteristics

\begin{tabular}{ll}
\hline Study characteristics & Experimental studies (e.g., RCT's) \\
\hline Study design & Quasi-experimental studies \\
& Observational studies when used to complement results from the experimental studies \\
Population & All medical conditions will be included as long as the intervention design uses gamification strategies \\
Interventions & Any intervention using gamification strategies will be considered \\
& Investigations focused on the efficacy or effectiveness of interventions (e.g., medical domain, educational domain) \\
& using gamification strategies will be included \\
Context & All countries and settings will be included. Specifically, clinical settings, educational settings, or rehabilitation settings
\end{tabular}

undertaken in the databases as follows: Scopus, IEEE, Web of Science, MEDLINE, ERIC, and PsycINFO. The databases were included with the consideration of the scope. To complement this research, authors investigating the topic of gamification will be contacted and asked help to identify studies that are not selected in the research or studies in press. In addition, the references of the articles included will be checked with the same aim. This search will be conducted independently by two reviewers.

\section{Search strategy}

This research will be carried out through the following expression: gamification OR "gam" element" "gam" based" AND intervention. Database searches will not have a limit range regarding the years of publication. The search terms will be used for all fields (including title, abstract, keywords, and full text), and all results will be included. All studies will be coded independently by a member of the research team. Afterwards, a second reviewer will work independently to undertake a quality appraisal considering the inclusion criteria. If necessary, the disagreements will be resolved in a discussion with a third party. It should be noted that reviewers will not be blind to the titles of the papers or to their authors.

Finally, only studies meeting predetermined quality criteria will advance to data extraction.

\section{Data management}

All records identified will be uploaded via EndNote X8 as a reference management software (Thomson Reuters, New York City, NY). This way, authors will easily identify any duplicates. Furthermore, this strategy will facilitate the exclusion ratios throughout the phases of the systematic review. Lastly, it will help build the diagram (i.e. PRISMA).

\section{Selection process}

In the Identification and Screening phases to assess the eligibility for inclusion in the systematic review, the titles and abstracts will be screened by two reviewers independently. After the selection by abstract, the articles in full-text will be checked to select those eligible for the systematic review. When unpublished or ongoing articles are identified, authors will be contacted and invited to share the information to be included in the review.

\section{Data collection process}

The two reviewers will independently extract the important information from the selected publications for the present review. The information extracted, mainly from the method and results sections, will analyze as follows: (i) type of intervention, (ii) participants, (iii) assessment, and (iv) results. Additionally, information about the gamification strategies used in each intervention, as well as relevant information of the intervention and assessment protocol will be collected. To minimize bias and improve the reliability of findings, paper scoring will be performed by the same researchers that analyzed and selected the paper and their relevant information. These two researchers will work independently; as a result of their work, papers found to meet the inclusion criteria will be eligible for the review. The results extracted will be summarized in accordance with the objectives of the study. Finally, the end report will be evaluated according to the PRISMA standards [22].

\section{Results}

Expected results are as follows: a list of the gamification strategies used (e.g., leaderboards, rankings), data on the efficacy of the gamification strategies used, impact of the use of the gamification strategies on the therapeutic involvement, and outcomes of the intervention (e.g., stable insulin levels in diabetic patients).

Additionally, systematization of the intervention characteristics using gamification strategies (e.g., duration, context of the intervention) and the main domains in which these strategies are used (e.g., medical, educational or rehabilitation domain). 


\section{Quality of evidence and risk of bias}

Each study included will be scored by two investigators according to the quality criteria for articles. This process will rely upon the use of tools developed to assess the internal validity of the studies. For example, the Cochrane Effective Practice and Organization of Care Risk of Bias Tool, developed to assess risk of bias of experimental and quasi-experimental studies, will be used. Another example is the BEME quality indicators (e.g., A risk of bias assessment is included in the manuscript?) [23]. This quality assessment will be carried out by two reviewers, and disagreements will be resolved appealing to a third reviewer through team discussion. Only studies with a positive assessment will be included in the systematic review.

\section{Data synthesis}

Authors will provide a narrative synthesis of the findings reported by the included studies. Results of the present investigation will be organized, among other aspects, according to the domain, type, and content of the intervention, the participants/patients' characteristics, the main results, the methodological quality appraisal, and the risk of bias assessment. To summarize the results, all data extracted will be synthesized using tables and graphs. The results will be organized by chronological order and the authors of the papers will be contacted whenever information is missing. When possible, the current authors will provide summaries of the intervention effects for each study by calculating risk ratios (for dichotomous outcomes) or standardized mean differences (for continuous outcomes). The present authors anticipate that there will be limited scope for running a meta-analysis because of the lack of standardization across studies regarding the intervention protocols, measures used, and sample. Finally, the evidence of publication bias will be assessed.

\section{Data reporting}

According to the PRISMA-P recommendations, the protocol was registered in the PROSPERO database. The systematic review will follow Cochrane's recommendations and will be reported based on PRISMA guidelines.

\section{Discussion}

This review will provide a synthesis of the extant literature on interventions using gamification' strategies. Moreover, the impact of using distinct elements of gamification on outcomes will also be examined. This review will be conducted based on strict and stringent guidelines that assures the quality of the review. Based on the results, current authors will be able to systematize the body of knowledge on this domain and provide clear guidelines for practitioners interested in intervening using gamification strategies. In addition, lines for future research likely to address gaps in the literature will be suggested. Furthermore, we believe that faculty and practitioners would benefit from learning about a set of gamification strategies that are efficacious irrespective of the context or populations investigated. This corpus of knowledge is expected to be transferred and used to inform high quality interventions.

\section{Limitations}

Regarding the limitations of the review, we can anticipate the publication bias as well as the language in which the article is published, since only articles written in English, Spanish and Portuguese are included.

\section{Additional file}

Additional file 1. The PRISMA-P checklist used while drafting the protocol manuscript.

\section{Abbreviations}

PRISMA: Preferred Reporting Items for Systematic Reviews and Meta-Analysis; PRISMA-P: Preferred Reporting Items for Systematic Reviews and Meta-Analysis-Protocols; RCT: Randomized Clinical Trials.

\section{Authors' contributions}

$\mathrm{SL}$ is the guarantor and provided methodological expertise. SL, AP and AO were responsible for the blinding literature search and the data extraction. PM oversaw data interpretation and provided technical guidance. PR made important intellectual contributions in the research design and manuscript revision. All authors provided input on the study design and wrote the drafts of the manuscript. All authors read and approved the final manuscript.

\section{Acknowledgements}

Authors would like to thank Sofia Kirkman for the English editing of the manuscript.

\section{Competing interests}

The authors declare that they have no competing interests.

Availability of data and materials

In this manuscript, the availability of data and material was not applicable.

\section{Consent for publication}

Not applicable.

Ethics approval and consent to participate

In this manuscript, ethics approval and consent to participate was not applicable.

\section{Funding}

This study was conducted at Psychology Research Centre (UID/ PSI/01662/2013), University of Minho, and supported by the Portuguese Foundation for Science and Technology and the Portuguese Ministry of Science, Technology and Higher Education through national funds and co-financed by FEDER through COMPETE2020 under the PT2020 Partnership Agreement (POCI-01-0145-FEDER-007653). Psychology Research Centre provided data-

base access to run this systematic review.

Sílvia Lopes (Grant No. SFRH/BD/118879/2016) and Armanda Pereira (Grant No. SFRH/BD/95104/2013) were supported by PhD fellowships 
from the Portuguese Foundation for Science and Technology (FCT). Paula Magalhães was supported by a Post-Doctoral fellowship from the Psychology Research Centre (CIPsi), University of Minho. These fellowships were granted to develop research within the gamification and rehabilitation domains, in which this systematic review is inserted.

\section{Publisher's Note}

Springer Nature remains neutral with regard to jurisdictional claims in published maps and institutional affiliations.

Received: 28 November 2018 Accepted: 19 February 2019

Published online: 22 February 2019

\section{References}

1. Deterding S, Dixon D, Khaled R, Nacke L. From game design elements to gamefulness. In: Proceedings of the 15th international academic MindTrek conference on envisioning future media environments-MindTrek'11. New York: ACM Press; 2011. http://dx.doi.org/10.1145/21810 37.2181040.

2. Attali Y, Arieli-Attali M. Gamification in assessment: do points affect test performance? Comput Educ. 2015;83:57-63. https://doi.org/10.1016/j. compedu.2014.12.

3. Cafazzo JA, Casselman M, Hamming N, Katzman DK, Palmert MR. Design of an mHealth app for the self-management of adolescent type 1 diabetes: a pilot study. J Med Internet Res. 2012;14(3):e70. https://doi. org/10.2196/jmir.2058.

4. Wolfe J, Chanin M. The integration of functional and strategic management skills in a business game learning environment. Simul Gaming. 1993;24(1):34-46. https://doi.org/10.1177/1046878193241005.

5. Xin C. Influence from the serious games on mobile game developers' commercial strategies. In: 2008 international seminar on business and information management. http://dx.doi.org/10.1109/ISBIM.2008.224.

6. Hamari J, Koivisto J, Sarsa H. Does gamification work? A literature review of empirical studies on gamification. In: 2014 47th Hawaii international conference on system sciences. New York: IEEE; 2014. http://dx.doi. org/10.1109/hicss.2014.377.

7. Elizabeth E, Jim L, Carol R, Liz S, Lindsey E, Arun T, et al. "Gamification" for health behaviour change in smartphone apps. Front Public Health. 2016. https://doi.org/10.3389/conf.fpubh.2016.01.00043.

8. Johnson D, Deterding S, Kuhn K-A, Staneva A, Stoyanov S, Hides L. Gamification for health and wellbeing: a systematic review of the literature. Internet Intervent. 2016;6:89-106. https://doi.org/10.1016/j.inven t.2016.10.002.

9. Hanus MD, Fox J. Assessing the effects of gamification in the classroom: a longitudinal study on intrinsic motivation, social comparison, satisfaction, effort, and academic performance. Comput Educ. 2015;80:152-61. https ://doi.org/10.1016/..compedu.2014.08.019.
10. Muessig KE, Nekkanti M, Bauermeister J, Bull S, Hightow-Weidman LB. A systematic review of recent smartphone, internet and web 2.0 interventions to address the HIV continuum of care. Curr HIV/AIDS Rep. 2015;12(1):173-90. https://doi.org/10.1007/s1 1904-014-0239-3.

11. Hertel NT, Vedel K, Rohde L, Olesen JB. Serious disease—serious game. Stud Health Technol Inform. 2013;192:1166. https://doi.org/10.3233/9781-61499-289-9-1166.

12. Kamel Boulos MN, Gammon S, Dixon MC, MacRury SM, Fergusson MJ, Miranda Rodrigues F, et al. Digital games for type 1 and type 2 diabetes: underpinning theory with three illustrative examples. JMIR Serious Games. 2015;3(1):e3. https://doi.org/10.2196/games.3930.

13. González-Gonzáleza CS, Toledo-Delgado P, Padrón M, Santos E, Cairos M. Including gamification techniques in the design of TANGO: H platform. Jurnal Teknologi. 2013. https://doi.org/10.11113/jt.v63.1958.

14. Browne K, Anand C, Gosse E. Gamification and serious game approaches for adult literacy tablet software. Entertain Comput. 2014;5(3):135-46. https://doi.org/10.1016/j.entcom.2014.04.003.

15. Landers RN. Developing a theory of gamified learning: linking serious games and gamification of learning. Simul Gaming. 2014;45(6):752-68. https://doi.org/10.1177/1046878114563660.

16. Starks K. Cognitive behavioral game design: a unified model for designing serious games. Front Psychol. 2014. https://doi.org/10.3389/fpsyg 2014.00028.

17. Brox E, Fernandez-Luque L, Tollefsen T, Brox E, Fernandez-Luque L, Tøllefsen T. Healthy gaming - video game design to promote health. Appl Clin Inform. 2011;2(2):128-42. https://doi.org/10.4338/aci-2010-10-r-0060.

18. Saeborn K, Fels D. Gamification in theory and action: a survey. Int J Hum Comput Stud. 2015;74:14-31. https://doi.org/10.1016/j.jihcs.2014.09.006.

19. Rojas D, Kapralos B, Dubrowski A. The missing piece in the gamification puzzle. In: Proceedings of the first international conference on gameful design, research, and applications-Gamification'13. New York: ACM Press; 2013. http://dx.doi.org/10.1145/2583008.2583033.

20. Moher D, Shamseer L, Clarke M, Ghersi D, Liberati A, Petticrew M, Shakelle P, Stewart L: Reporting guidelines for systematic review protocols. In: 19th Cochrane Colloquium; October 19-22. Madrid, Spain; 2011.

21. Higgins JP, Green S. Guide to the contents of a cochrane protocol and review. Cochrane handbook for systematic reviews of interventions. Hoboken: John Wiley \& Sons Ltd; 2008. p. 51-79. https://doi. org/10.1002/9780470712184.ch4.

22. Moher D, Liberati A, Tetzlaff J, Altman DG. Preferred reporting items for systematic reviews and meta-analyses: the PRISMA statement. BMJ. 2009;339:b2535. https://doi.org/10.1136/bmj.b2535.

23. Buckley S, Coleman J, Davison I, Khan KS, Zamora J, Malick S, Morley D, Pollard D, Ashcroft T, Povic C, Sayers J. The educational effects of portfolios on undergraduate student learning: a best evidence medical education (BEME) systematic review. BEME guide no. 11. Med Teach. 2009;31(4):282-98.

\footnotetext{
Ready to submit your research? Choose BMC and benefit from:

- fast, convenient online submission

- thorough peer review by experienced researchers in your field

- rapid publication on acceptance

- support for research data, including large and complex data types

- gold Open Access which fosters wider collaboration and increased citations

- maximum visibility for your research: over $100 \mathrm{M}$ website views per year
}

At BMC, research is always in progress.

Learn more biomedcentral.com/submissions 